\title{
Medication Home Storing and Self Drug Prescriptionas Part of Inappropriate Use of Pharmaceuticals Among Families in Hail - Saudi Arabia
}

\author{
Naif Mohammed Altamimi
}

\begin{abstract}
In many parts of the world up to $80 \%$ of illness episodes are self-treated with modern medications. Public health might be affected by inappropriate use of medication including:polypharmacy, inappropriate dose alteration, Failure to prescribe in context with clinical guidelines and Self-prescriptions of medications. Aims: To investigate the magnitude of medication home storing and self drug prescription and common inappropriate use of medication among Hail, Saudi Arabia. Settings and Design: Cross Sectional Study. Methods and Material: A questionnaire was distributed to 374 participants among different families in Hail,Saudi Arabia. Asking individualsabout wheather they practice the common Inappropriate uses of medication or not. Results:The study revealed that $41 \%$ of people tend to store drugs at home, while $29 \%$ of cases had the habit of getting rid of it. $40 \%$ of people self prescribe medication. $22 \%$ of people increase the dose of the drug when they do not feel better while 60\% are committed with the dose. 57\% of people stopped using the drugs after they are clinically improved without following the Instructions of Physician. Conclusions: Inappropriate use of medication is a rising problem in modern public health. People have the practice of self drug prescription and medication storing at home. Others may not be commited to physician advice. These inappropriate use may affect public health from different prospectives. People need to be aware of the effect of inappropriate use of Pharmaceuticals on patient and society.
\end{abstract}

Keywords: Pharmaceuticals, Home Storing, Self Prescription

Key Messages: Medication home storing and self-drug prescriptions may cause a major drug interaction and inappropriate use of Antibiotics can raise the problem of Bacterial Resistant. Investigating the magnitude is necessary to make appropriate Intervention to increase awareness among community.

\section{Introduction}

Medication that may be given to people either precribed or over the counter may cause an effect on the general society health if used inappropriately.

People need information and education on medications and appropriate and effective treatment seek strategies because of the huge role of pharmaceuticals in modern health care; individuals own health responsibility, perfect therapeutic regimens, \& human rights.

A lot of studies take the misconception and the misuse of non medical drug prescribers, pharmacist and consumers. Pharmaceutical marketing may also contribute to the misuse of it.

In many parts of the world up to $80 \%$ of illness episodes are self-treated with modern medications. Even when patient use usual health care provider, their choice is the ultimate determinant of drugs use. These choices are affected by the beliefs of family or friends or the larger community, information from health providers, pharmacists, and promotional material. How medicines are obtained and used is therefore not just about individual choices made by a prescriber or consumer but reflects a core of societal, economic and health factors which influence those decisions.

Common types of inappropriate use of pharmaceuticals are: Patient polypharmacy, Inappropriate use of antibiotics in cases of non-bacterial cause or inappropriate dose alteration,
Failure to prescribe in context with clinical guidelines and Self-prescriptions of medications.

This study aims to investigate the magnitude of medication home storing and Self-drug prescription among people in the absence of strict roles to pharmacies in the society of Hail, Saudi Arabia. Some pharmacies might be able to give an antibiotic without a prescription, which may cause bacterial resistent if it is not used in the proper dose or Indication. one of our objectives was to investigate the bacterial resistance to one of the most common antibiotics prescribed "Amoxicillin"

\section{Subjects and Methods}

A questionnaire was distributed to 374 participants among different families in Hail,Saudi Arabia. Asking individuals wheather they have a private pharmacy at home and how often they use the medication again without medical advice from physician. Subjects also asked about wheather they listen to family and friends advice about medication and if they ever self prescribed some medication. Subjects also has been asked if they stop completing the medication as adviced if they feel clinically improved. Also they have been asked how often they read the medication sheet pefore taking theire medications. Mouth swaps obtained after Permission from 19 patients with a documented upper respiratory tract infection (URTI) following prescription of Amoxicillin antibiotic as a treatment. Then, a bacterial cultures was done to monitor the efficacy of "Amoxicillin" by Antibiotic Sensitivity Test. 


\section{International Journal of Science and Research (IJSR) \\ ISSN (Online): 2319-7064}

Index Copernicus Value (2013): 6.14 | Impact Factor (2014): 5.611

\section{Results}

The age of participants range from 20-40 years. The study revealed that $41 \%$ of people tend to keep drugs at home to use them again according to the needs, while $29 \%$ of cases had the habit of getting rid of it. $40 \%$ of people mentioned the tradition use self-prescription drugs. $22 \%$ percent of people increase the dose of the drug when they do not feel better while $60 \%$ are committed with the dose. $57 \%$ of people stopped using the drugs just after they are clinically improved without following the Instructions of Physician.Headache and cold contribute for about $46 \%$ of medicines that they tend to take without medical advice. Allergy, joint and muscle pain and abdominal colicky pain. Amoxicillin was an effective drug against almost all organisms detected in swaps cultures but with variable extent.

\section{Discussion}

The study revealed that $41 \%$ of people tend to keep drugs at home which makes the misuse of the medication is easy as soon as they develop the same symptoms again. Although most of the government hospitals usually give the patient the quantity of medication that they need to complete the course, $57 \%$ of people in the Study do not complete the whole course and stop as soon as they feel better. only $29 \%$ of peopleget rid of the remaining medication if the hospital gave them more than what they need.

$40 \%$ of people use self-prescription drugs. Based on their limited knowledge of medicine and previous experiences with diseases. $22 \%$ percent of people increase the dose of the drug when they do not feel better which might raise the possibilities of medication toxicity and side effects.

Most of the samples that was taken and cultured from the patient was sensitive to Amoxicillin Antibiotic. Although it was a small samples but more large study sample is needed to investigate the sensitivity and probably detect some antibiotic resistance due to the common inappropriate use of antibiotic in the society. Awareness campaigns is important to raise the level of awareness in the society about appropriate use of medication and complications that they might be suscebtiple to.

\section{Conclusions}

Inappropriate use of medication is a rising problem in modern public health. People have the practice of self drug prescription and medication storing at home. Others may not be commited to physician advice. These inappropriate use may affect public health from different prospectives. People need to be aware of the effect of inappropriate use of Pharmaceuticals on patient and society.

\section{References}

[1] YenenehH ,Gyorkos TW, Joseph L , Pickering J , Tedia S. Antimalarial Drug Utilization by Women in Ethiopia: a knowledge-attitudes-practice study. Bulletin of the World Health Organization, 1993. 71 (6): 763-772)
[2] Dua V, Kunin CM, White LV. The Use of Antimicrobial Drugs in Nagpur, India: a window on medical care in a developing country. SocSci Med 1994; (38): 717-724

[3] Mwenesi H, Harpham T, Snow RW. Child Malaria Treatment Practices among Mothers in Kenya. Soc. Sci. Med 1995; 40 (9): 1271-1277

[4] KiyingiKS ,Lauwo JAK . Drugs in the Home : danger and waste. World Health Forum 1993; 14: 381-416

[5] Hardon A, van der Geest S. Hazards of Self-Medication. World Health Forum 1987; 8: 469-471

[6] Fabricant SJ, Hirschhorn N. Deranged Distribution, Perverse Prescription, Unprotected Use: the irrationality of pharmaceuticals in the developing world. Health Policy and Planning 1987; 2(3):204-213.

[7] Schwartz RK ,Soumerai SB , Avorn J. Physician Motivations For Nonscientific Drug Prescribing. SocSciMed . 1989; 28: 577-582 